\title{
THE EQUIVARIAN'T PLATEAU PROBLEM AND INTERIOR REGULARITY
}

\author{
BY
}

\author{
H. BLAINE LAWSON, JR.
}

\begin{abstract}
Let $M \subset \mathbf{R}^{n}$ be a compact submanifold of Euclidean space which is invariant by a compact group $G \subset S O(n)$. When $\operatorname{dim}(M)=n-2$, it is shown that there always exists a solution to the Plateau problem for $M$ which is invario ant by $G$ and, furthermore, that uniqueness of this solution among $G$ invariant currents implies uniqueness in general. This result motivates the subsequent study of the Plateau problem for $M$ within the class of $G$-invariant integral currents. It is shown that this equivariant problem reduces to the study of a corresponding Plateau problem in the orbit space $\mathrm{R} / G$ where, for "big" groups, questions of uniqueness and regularity are simplified. The method is then applied to prove that for a constellation of explicit manifolds $M$, the cone $C(M)=\{t x ; x \in M$ and $0 \leq t \leq$ 1 ) is the unique solution to the Plateau problem for $M$. (Thus, there is no hope for general interior regularity of solutions in codimension one.) These manifolds ino clude the original examples of type $S^{n} \times S^{n} \subset \mathrm{R}^{2 n+2}, n \geq 3$, due to Bombieri, DeGiorgi, Giusti and Simons. They also include a new example in $R^{8}$ and examples in $\mathbf{R}^{n}$ for $n \geq 10$ with any prescribed Betti number nonzero.
\end{abstract}

1. Introduction. Let $G$ be a compact, connected Lie group acting orthogonally on $\mathbf{R}^{n}$, and let $M$ be a compact, oriented, $(n-2)$-dimensional, $G$-invariant manifold, of class $C^{k}$, imbedded in $S^{n-1}=\left\{x \in \mathbf{R}^{n}:|x|=1\right\}$. By a solution to the pla. teau problem for $M$ in $\mathbf{R}^{n}$ we mean an $(n-1)$-dimensional integral current $T$ with $\partial T=M$ whose mass $M(T)$ satisfies $M(T)=\inf \left\{M\left(T^{0}\right): T^{\prime}\right.$ is an integral current in $\mathbf{R}^{n}$ and $\left.\partial T^{\prime}-M\right\}$. The existence of $T$ follows from general theory (and uniqueness does not, in general, hold). The support of $T$ minus another compact set $\mathcal{S}$ whose Hausdorff dimension does not exceed $n-8$ is an $(n-1)$-dimensional analytic manifold [5] and [7]. Hence, if $n \leq 7$, then $\mathcal{S}=\varnothing$. If $n=8, \mathcal{S}$ consists of at most isolated points $[5,5.4 .16]$. Moreover, for any $n$ we know from [1] that near $M$ the support of $T$ has the structure of a regular manifold with ( $M$ as) boundary.

In the case where $M$ possesses certain symmetries as above it is natural to investigate the possible symmetries of $T$. If the condition that $G$ be connected is dropped, it is not necessarily true that every solution to the Plateau problem for $M$ is $G$-invariant [5, 5.4.17]. However, we shall show that whenever $G \subset \operatorname{SO}(n)$, there always exists at least one invariant solution and, moreover, that uniqueness

Received by the editors April 15, 1970 and, in revised form, September 20, 1971. AMS (MOS) subject classifications (1970). Primary 49F 22, 49F 20; Secondary 35D10, 28A75, 58A25.

Key words and phrases. Plateau problem, integral current, Goinvariant current, minimal cone, interior regularity. 
in the class of Goinvariant integral currents implies uniqueness in general.

The next step is to study the Plateau problem for $M$ within the class of $G$-invariant integral currents. We show how this reduces to the study of a corresponding Plateau problem in the space $\mathbf{R}^{n} / G$ with an appropriate metric structure (cf. [8]) where in some cases the questions of uniqueness and regularity of solutions are vastly simplified. In particular, for many cases the problem is reduced to one of studying the geodesics on a certain Riemannian manifold.

As an application, we then give a new proof that the cone

$$
C\left(S^{n} \times S^{n}\right)=\left\{(x, y) \in \mathbf{R}^{n+1} \times \mathbf{R}^{n+1}:|x|^{2} \leq|y|^{2}=1 / 2\right\}
$$

is the unique solution to the Plateau problem for $S^{n} \times S^{n} \subset \mathbf{R}^{2 n+2}$ (first proved by Bombieri, DeGiorgi and Guisti [3]). This result dashes the hope for general interior regularity of (codimension-one) solutions to the Plateau problem in $\mathbf{R}^{8}$. The method here, however, is sufficiently general that with a uniform treatment we produce many more minimizing cones of different topological types even in dimension 8. Furthermore, by studying the geodesic structure of certain orbit spaces one can get a clear geometric picture of why interior regularity eventually breaks down.

I would like to express gratitude to Wu-YiHsiang for invaluable help in the development of these ideas and to Wendell Fleming for suggesting a much more elegant proof of Theorem 1. I also wish to thank Herbert Federer and John Brothers for many helpful suggestions.

2. Notation. Let $X$ be a Riemannian manifold. We denote by $E^{k}(X)$ the space of smooth, exterior $k$ forms on $X$ with the usual topology. We let $E_{k}(X)$ be its dual space and denote by $I_{k}(X) \subset E_{k}(X)$ the subgroup of $k$-dimensional integral currents (with compact support) in $X$ (see [6]). For each $k$ there is a boundary map $\partial_{:} E_{k}(X) \rightarrow E_{k-1}(X)$ defined by setting $(\partial T)(\phi)=T(d \phi)$ for $T \in E_{k}(X)$ and $\phi \in E^{k-1}(X)$ where $d: E^{k-1}(X) \rightarrow E^{k}(X)$ is exterior differentiation.

At each point $p$ of $X$ we introduce a norm $\|\cdot\|$ on $\Lambda^{k} X_{p}$ by setting

$$
\|v\|=\inf \left\{\sum_{i}\left|v_{i}\right|: v=\sum v_{i} \text { and each } v_{i} \text { is a simple vector }\right\}
$$

where $|\cdot|$ is the usual norm obtained from the metric on $X_{p}$. For $\phi \in E^{k}(X)$ we define the mass of $\phi$ to be $M(\phi)=\sup \left\{\left\|\phi_{p}\right\|: p \in X\right\}$. For $T \in E_{k}(X)$ we then define the mass of $T$ as $M(T)=\sup \{T \phi: M(\phi) \leq 1\}$. Associated to each $T \in E_{k}(X)$ for which $M(T)<\infty$ we have the variation measure $\|T\|$ of $T$ defined by setting $\|T\|(f)$ $=\sup \{T \phi:\|\phi\| \leq f\}$ for each nonnegative, real valued, continuous function $f$ on $X$.

For any locally Lipschitzian map $f_{:} X \rightarrow X^{\prime}$ where $X^{\prime}$ is another Riemannian manifold, we denote the induced map on currents by $f_{H^{*}} E_{*}(X) \rightarrow E_{*}\left(X^{0}\right)$. 
For any notation and terminology not mentioned here the reader is referred to [5] and [6].

3. The existence of invariant, minimizing currents. Let $M$ and $G$ be as above and suppose we have $T \in I_{n-1}\left(\mathbf{R}^{n}\right)$ such that $\partial T=M$ (where $M$ is now interpreted as an integral current) and such that $M(T)=\inf \left\{M\left(T^{\prime}\right): T^{\prime} \in I_{n-1}\left(\mathbf{R}^{n}\right)\right.$ and $\left.\partial T^{\prime \prime}=M\right\}$. The existence of $T$ is guaranteed by 9.6 in [6]. From this $T$ we will construct a $G$-invariant current having mass $M(T)$ and boundary $M$. The following elegant proof was shown to us by Wendell Fleming and is based on an idea of K. Krickeberg.

Theorem 1 (W. Fleming). There exists a family $\left\{T_{\lambda}\right\}_{0 \leq \lambda \leq 1}$ of G-invariant in. tegral currents in $\mathbf{R}^{n}$ sucb that

$$
\int_{0}^{1} T_{\lambda} d \lambda=\int_{G} g_{\#} T d g
$$

(where dg represents Haar measure normalized so the measure of $G$ is 1 ), and for almost all $\lambda$ we have $\partial T_{\lambda}=M$ and $M\left(T_{\lambda}\right)=M(T)$.

Proof. Consider the $G$-invariant $(n-1)$-dimensional normal current

$$
\widetilde{T}=\int_{G}\left(g_{\#} T\right) d g .
$$

Since $\partial g_{\#} T=M$ for all $g \in G$ we have $\partial \widetilde{T}=M$, and, by the lower semicontinuity of the mass function, $M(\widetilde{T}) \leq M(T)$. Our first step shall be to represent $T$ as part of the boundary of an $n \cdot$ dimensional integral current.

Let $S^{n-1}=\left\{x \in \mathbf{R}^{n}:|x|=1\right\}$ and recall that $M$ is a compact oriented $(n-2)$ dimensional manifold embedded in $S^{n-1}$. Hence, $M$ divides $S^{n-1}$ into two components $D$ and $D^{\prime}$. Orient $D$ and $D^{\prime}$ by the exterior unit normal to $S^{n-1}$ and arrange the notation so that $\partial D=-M$. Since $G$ is connected and leaves both $M$ and $S^{n-1}$ invariant, it must also leave $D$ invariant. Since $\partial(T+D)=0$, there exists a unique current $U \in I_{n}\left(\mathbf{R}^{n}\right)$ such that $\partial U=T+D$. (See $[6,5.11]_{0}$ ). Associated to $U$ is an integer valued function $f$ with support in $B^{n}=\left\{x \in \mathbf{R}^{n}:|x| \leq 1\right\}$ such that for

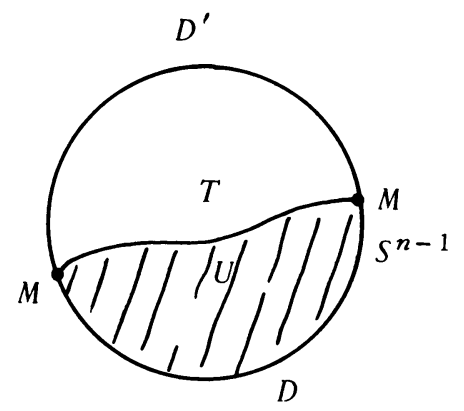

any smooth function $\phi$ with compact support on $\mathbf{R}^{n}$ we have

$$
U\left(\phi d x_{1} \wedge \ldots \wedge d x_{n}\right)=\int_{\mathrm{R}^{n}} \phi f d x
$$


We denote this relationship by writing $U=c f$. (Note. supp $U \subset B^{n}$ because supp $\partial U \subset B^{n}$.)

Since $T$ minimizes mass, its support has finite Hausdorff $(n-1)$-measure。 Moreover, we have

$$
\text { supp } T \cap S^{n-1}=\operatorname{supp} M .
$$

From 2.3 in [6] we see that $f$ is constant on each component of $B^{n} \sim$ (supp $T U$ $\left.S^{n-1}\right)$. We assert that

(a) $f=1$ on the component whose boundary contains $D$,

(b) $f=0$ on the component whose boundary contains $D^{\prime}$,

(c) $f(x)=0$ or 1 for all $x$ 。

To prove assertion (a) choose a small ball $B$ which is centered in $D$ and does not meet supp $T$. Then $\partial(U \cap B)=D \cap B+k B^{n} \cap(\partial B)$ and since $0=\partial^{2}(U \cap B)$ we have $k=1$.

For assertion (c) we write $f$ as a sum of functions $f=f_{1}+f_{2}$ where $f_{1}(x)=1$ if $f(x)=1$ and $f_{1}(x)=0$ otherwise. Let $U_{1}=c f_{1}$ and $U_{2}=c f_{2}$ be the associated currents. From the total gradient variational formula $[5,4.5 .9(13)]$ we have

$$
M(\partial U)=M\left(\partial U_{1}\right)+M\left(\partial U_{2}\right) .
$$

Moreover, we have

$$
\partial U=T+D, \quad \operatorname{supp} T \cap \operatorname{supp} D=\operatorname{supp} M
$$

and

$$
\partial U_{1}=T_{1}+D, \quad \operatorname{supp} T_{1} \cap \operatorname{supp} D=\operatorname{supp} M .
$$

Since supp $M$ has dimension $n-2$, it follows that $M(\partial U)=M(T)+M(D)$ and $M\left(\partial U_{1}\right)=M\left(T_{1}\right)+M(D)$, and therefore that $M(T) \geq M\left(T_{1}\right)$. However, $\partial T=\partial T T_{1}$ and $T$ is minimizing. Hence, $M(T)=M\left(T_{1}\right)$ and thus $M\left(\partial U_{2}\right)=0$. Finally, $\partial U_{2}=0 \mathrm{im}$ plies that $U_{2}=0$ because $U_{2}$ has codimension zero. Thus $U=U_{1}, f=f_{1}$, and the assertion is proved.

We now define a function $\tilde{f}(x)=\int_{G} f(g x) d g$ with associated current

$$
\widetilde{U}=\int_{G}\left(g_{\#} U\right) d g=c \tilde{f} \text {. }
$$

Note that $0 \leq \tilde{f} \leq 1$ and $\partial \tilde{U}=\tilde{T}+D$. For $\lambda \in[0,1]$ we define $\chi_{\lambda}$ to be the characteristic function of the set $E_{\lambda}=\left\{x \in \mathbf{R}^{n}: \tilde{f}(x)>\lambda\right\}$. Let $\tilde{U}_{\lambda}=c \chi_{\lambda} \cdot\left(\tilde{U}_{\lambda}\right.$ is simply the set $\tilde{U}_{\lambda}$ with positive orientation interpreted as a current.) From the fact that $\tilde{f}=\int_{0}^{1} \bar{\chi}_{\lambda} d \lambda$ we have that

$$
\widetilde{U}=\int_{0}^{1} \tilde{U}_{\lambda} d \lambda \text { and } \partial \tilde{U}=\int_{0}^{1} \partial \tilde{U}_{\lambda} d \lambda
$$


From the total gradient variational formula $[5,4.5 .9(13)]$ it follows that

$$
M(\partial \tilde{U})=\int_{0}^{1} M\left(\partial \tilde{U}_{\lambda}\right) d \lambda .
$$

From the remarks made while proving assertion (a) above we see that any point of $D$ has a neighborhood $B$ such that $\widetilde{f}(x)=1$ on $B \cap$ (interior of $B^{n}$ ). Therefore, $B \cap$ (interior of $B^{n}$ ) $\subset E_{\lambda}$ for $\lambda<1$. Hence, writing

$$
\partial \tilde{U}=\tilde{T}+D, \quad \partial \widetilde{U}_{\lambda}=\widetilde{T}_{\lambda}+D, \quad 0<\lambda<1,
$$

we have

$$
\operatorname{supp} \tilde{T} \cap \operatorname{supp} D=\operatorname{supp} M, \quad \operatorname{supp} \widetilde{T}_{\lambda} \cap \operatorname{supp} D=\operatorname{supp} M,
$$

and thus

$$
M(\partial \tilde{U})=M(\tilde{T})+M(D), \quad M\left(\partial \tilde{U}_{\lambda}\right)=M\left(\tilde{T}_{\lambda}\right)+M(D)
$$

It then follows from (3.1) that

$$
M(\tilde{T})=\int_{0}^{1} M\left(\tilde{T}_{\lambda}\right) d \lambda
$$

Clearly we have that

$$
\widetilde{T}=\int_{0}^{1} \widetilde{T}_{\lambda} d \lambda, \quad \partial \widetilde{T}=\partial \widetilde{T}_{\lambda}=M, \quad \text { and } \quad g_{\#} T_{\lambda}=T_{\lambda}
$$

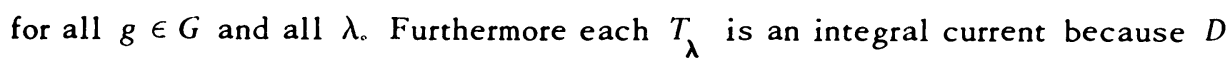
and $\partial U_{\lambda}$ are.

Since $T$ is minimizing and $M(\tilde{T})<M(T)$ we have $M\left(\widetilde{T}_{\lambda}\right)=M(T)$ for almost all $\lambda$. This completes the proof.

Theorem 1 asserts the existence of $G$-invariant solutions to the Plateau problem and, upon close inspection, also gives the following uniqueness result.

Corollary 3.1. If the solution to the Plateau problem for $M$ is unique in the class of G-invariant currents, it is unique in general.

Proof. Let $T^{*}$ be the unique invariant solution, and $\underset{\sim}{\sim} T, U, T_{\lambda}$, etc be as above. Then $T_{\lambda}=T^{*}$ for $0<\lambda<1$. Hence $\widetilde{T}=T^{*}$, and $\tilde{f}(x)=0$ or 1 for all $x$. Thus $f=\tilde{f}$ which implies $U=\widetilde{U}$ and $T=\widetilde{T}=T^{*}$.

Remark 3.2. It has been pointed out by John Brothers that Theorem 1 remains true when $G$ is any compact subgroup of $S O(n)$. This follows from the fact that since $g_{\sharp} M=M$ we have either $g_{\#} D=D$ or $g_{\#} D=-D^{\prime}$. In the second event $g$ cannot be in $S O(n)$.

Remark 3.3. The fact that $G \subset S O(n)$ is essential for Theorem 1. To see this consider the boundary $S^{1} \times S^{1}=\left\{(z, w) \in \mathbf{C} \times \mathbf{C}:|z|^{2}=|w|^{2}=1 / 2\right\} \subset \mathbf{C}^{2}=\mathbf{R}^{4}$ and 
the group $\mathbf{Z}_{2} \subset O(4)$ generated by $f: \mathbf{C}^{2} \rightarrow \mathbf{C}^{2}$ where $f(z, w)=(w, \bar{z})(\mathbf{C}=$ complex numbers). The solutions to the Plateau problem for $S^{1} \times S^{1}$ are not $\mathbf{Z}_{2}$-invariant. There are exactly two distinct solutions (which are images of each other under $Z_{2}$ ). For details of this case see $\$ 5$ and $[5,5.4 .17]$.

4. Reduction of the equivariant problem. Our purpose here is to reduce the Plateau problem for $G$ invariant currents to a corresponding problem in the space of orbits. We begin with the case which is simplest geometrically.

Let $X$ be an $(n+p)$-dimensional oriented Riemannian manifold and $G$ a compact, connected group of isometries of $X$ (a sub-Lie group of $I S O(X)$ )。 We shall assume for the moment that all the orbits of $G$ on $X$ are of the same type (i.e., all the isotropy subgroups $G_{x}=\left\{g \in G_{:} g x=x\right\}$ for $x \in X$ are mutually conjugate in $G)$. Then there is a natural differentiable structure on the space of orbits $X / G$ so that $G / G_{x} \rightarrow X \stackrel{\pi}{\rightarrow} X / G$ is a differentiable fibre bundle. Moreover, the manifold $X / G$ also has a natural Riemannian metric $\langle\cdot, \cdot\rangle$ such that if $\xi$ and $\eta$ are tangent vectors at $x \in X$, which are orthogonal to the orbit $G(x)$, then $\langle\xi, \eta\rangle=\left\langle\pi_{*} \xi, \pi_{*} \eta\right\rangle$ 。 With this metric $\pi$ is a Riemannian submersion in the sense of [9]. The length of a curve in $X / G$ is the length of any orthogonal trajectory through the corresponding orbits in $X$ 。

Let $p$ denote the common dimension of the orbits of $G$ on $X$ and define the volume function $V: X / G \rightarrow \mathbf{R}$ by setting $V(x)=p$-dimensional volume of $\pi^{-1}(x)$. (For convenience we shall also denote the function $\pi^{*} V$ on $X$ by $V_{0}$ ) There are several other Riemannian metrics on $X$ which will be of interest here. For each integer $k, 1 \leq k \leq \operatorname{dim} X / G$, we define the metric $\langle\cdot, \cdot\rangle_{k}$ on $X / G$ by

$$
\langle\cdot, \cdot\rangle_{k}=V^{2 / k}\langle\cdot, \cdot\rangle \cdot
$$

Then the area of a $k$-dimensional submanifold of $X / G$ in the metric $\langle\cdot, \cdot\rangle_{k}$ is equal to the area of its inverse image in $X$.

A current $T$ is called $G$-invariant if $g_{\#} T=T$ for all $g \in G$. We denote by $I_{k}^{G}(X)$ the space of $G$-invariant, $k$-dimensional integral currents in $X$ (if $k<p$, $\left.I_{k}^{G}(X)=0\right)$, and define a continuous $\mathbf{Z}$-linear map

$$
\pi_{*}: I_{*}^{G}(X) \rightarrow I_{*}(X / G)
$$

of degree $-p$ by setting

$$
\left(\pi_{*} T\right)(\omega)=T\left(\pi^{*} \omega \wedge \Omega\right)
$$

for $T \in I_{k+p}^{G}(X)$ and $\omega \in E^{k}(X / G)$ where $\Omega=(1 / V) \Omega_{0}$ and where $\Omega_{0}$ is the field of positively oriented unit cotangent planes to the orbits of $G$ on $X$.

Theorem 2. The map $\pi_{*}$ bas the following properties:

(a) $\pi_{*}$ bas a continuous 2 -sided inverse of degree $p$ 


$$
\pi^{*}: I_{*}(X / G) \rightarrow I_{*}^{G}(X)
$$

such that if $M$ is a compact, oriented, k-dimensional, embedded submanifold (with boundary) of $X / G$ and if $\mathbf{M}$ is the current associated to $M$, then $\pi^{*}(\mathbf{M})=\pi^{-1}(\mathbf{M})$.

(b) $\partial \circ \pi_{*}=\pi_{*} \circ \partial$ (and $\partial \circ \pi^{*}=\pi^{*} \circ \partial$ ).

(c) If $X / G$ is given the metric $\langle\cdot, \cdot\rangle_{k}$ and if $M_{k}$ is the associated mass function, then $M(T)=M_{k}\left(\pi_{*} T\right)$ for all $T \in I_{p+k}^{G}(X)$.

Proof. The maps $\pi_{*}$ and $\pi^{*}$ were first considered by John Brothers and we refer to Theorems 3.3, 3.5, 3.6 and 3.7 in [4] for the proof of parts (a) and (b) above. We note that Theorem 3.3 (the statements of proof of which undergo a trivial modification to fit our needs here) also proves the fact that $\pi_{*}\left[I_{*}^{G}(X)\right] \subset$ $I_{*}(X / G)$ 。

For part (c) we make some preliminary observations. Let $T \in I_{p+k}^{G}(X)$. From basic facts concerning currents of finite mass (in [6]) we know there exists over $X$ a Borel measurable field of $(p+k)$-vectors $\vec{T}$ such that, for any $\omega \in E^{p+k}(X)$,

$$
T(\omega)=\int_{X} \omega(\vec{T}) d\|T\|,
$$

where $\|T\|$ is the variation measure associated to $T$. Since $T$ is $G$-invariant and rectifiable, it follows that $\vec{T}$ is $G$-invariant and that, for $\|T\|$ almost every $x \in X$, we have $\vec{T}=\vec{T}_{1} \wedge \vec{T}_{0}$, where $\vec{T}_{0}$ is the unit $p$-vector representing the tangent plane to the orbit at $x$ 。

We observe also that, for any $\omega \in E^{p+k}(X), T(\phi)=T(\tilde{\phi})$ where $\tilde{\phi}=$ $\int_{G}\left(g^{*} \phi\right) d g$. Since $\tilde{\phi}$ is $G$-invariant, it may be expressed as $\tilde{\phi}=\pi^{*} \omega \wedge \Omega_{0}+\tilde{\phi}_{1}$ where $\omega \in E^{k}(X / G)$ and where $\widetilde{\phi}_{1}\left(\vec{T}_{1} \wedge \vec{T}_{0}\right) \equiv 0$ for any $k$-vector field $\vec{T}_{1}$.

If we now introduce the metric $\langle\cdot, \cdot\rangle_{k}$ on $X / G$ and denote by $M_{k}$ the corresponding mass norms on $E^{*}(X / G)$ and $I_{*}(X / G)$, we have for $T \in I_{p+k}^{G}(X)$ that

$$
\begin{aligned}
M_{k}\left(\pi_{*} T\right) & =\sup \left\{\left(\pi_{*} T\right)(\omega): \omega \in E^{k}(X / G) \text { and } M_{k}(\omega) \leq 1\right\} \\
& =\sup \left\{\left(\pi_{*} T\right)(\omega): \omega \in E^{k}(X / G) \text { and }\left\|\omega_{x}\right\| \leq V(x) \text { for all } x \in X / G\right\} \\
& =\sup \left\{T\left(\pi^{*} \omega \wedge \Omega\right): \omega \in E^{k}(X / G) \text { and }\left\|\omega_{x}\right\| \leq V(x) \text { for all } x \in X / G\right\} \\
& =\sup \left\{T\left(\pi^{*} \omega \wedge \Omega_{0}\right): \omega \in E^{k}(X / G) \text { and } M(\omega) \leq 1\right\}
\end{aligned}
$$

(where $\|\cdot\|$ is the norm defined by $(2.1)$ and associated to the canonical metric $\langle\cdot, \cdot\rangle)$. On the other hand, from the observations above, we have that

$$
\begin{aligned}
M(T) & =\sup \left\{T: \phi: \phi \in E^{p+k}(X) \text { and } M(\phi) \leq 1\right\} \\
& =\sup \left\{T \tilde{\phi}: \phi \in E^{p+k}(X) \text { and } M(\phi) \leq 1\right\} . \\
& =\sup \left\{T\left(\pi^{*} \omega \wedge \Omega_{0}\right):(1) \in E^{k}(X: G) \text { and } M(\omega) \leq 1\right\} .
\end{aligned}
$$

This proves (c) and completes the theorem. 
Remark. Part (b) of Theorem 2 can be verified directly as follows. Fix $T \in$ $I_{p+k}^{G}(X)$. It is clearly sufficient to prove that $T\left(\pi^{*} \omega \wedge d \Omega\right)=0$ for any $\omega \epsilon$ $E^{k-1}(X / G)$. This fact is an immediate consequence of the following two formulas which follow from straightforward computation.

$$
\begin{aligned}
d V & =-V K, \\
d \Omega_{0} & =-K \Omega_{0}+\Omega_{1},
\end{aligned}
$$

where $\Omega_{1}\left(\vec{T}_{1} \wedge \vec{T}_{0}\right) \equiv 0$ for any vector field $\vec{T}_{1}$ and where $\mathcal{K}$ denotes the mean curvature form for the orbits of $G$.

Theorem 2 has the following immediate corollary.

Corollary 4.1. Let $\bar{T}$ be an element of $I_{p+k}^{G}(X)$ and set $B=\partial \bar{T}$. Let $X / G$ be given the metric $\langle\cdot, \cdot\rangle_{k^{\circ}}$ Then the numbers

$$
m=\inf \left\{M(T): T \in I_{p+k}^{G}(X) \text { and } \partial T=B\right\}
$$

and

$$
m^{\prime}=\inf \left\{M\left(T^{\prime}\right): T^{\prime} \in I_{k}(X / G) \text { and } \partial T^{\prime}=\pi_{*} B\right\}
$$

are equal. Moreover, the map $\pi_{*}$ gives a one-to-one correspondence between cur. rents in $I_{p+k}^{G}(X)$ of least mass having $B$ as boundary and those in $I_{k}(X / G)$ of least mass having $\pi_{*} B$ as boundary.

We now return to the case of a compact, connected Lie group $G$ represented orthogonally on $\mathbf{R}^{n}$. The orbits of $G$ are, in general, not all of the same type. However, there is an open dense submanifold $X \subset \mathbf{R}^{n}$ which is comprised of orbits of the same type, the so-called principal orbits, and which fits into the setting above. We denote by $p$ the common dimension of the principal orbits, and to simplify the discussion we shall assume that the dimension of all nonprincipal orbits is $<p$, i.e., there are no "exceptional orbits." (This last condition can be dropped as in" dicated in Remark 4.5.) The singular set $S=\mathbf{R}^{n} \sim X$ is a differentiable stratified set (stratified by orbit types) of codimension at least two. The volume function $V: \mathbf{R}^{n} \rightarrow \mathbf{R}^{+} \cup\{0\}$ is then defined as follows:

$$
V(x)= \begin{cases}\operatorname{volume}[G(x)] & \text { if } x \in X, \\ 0 & \text { if } x \in S .\end{cases}
$$

It is not difficult to see that $V^{2}$ is an invariant polynomial of degree $2 p$.

The orbit space $R^{n} / G$ has the natural structure of a differentiable stratified set such that over each stratum the restriction of $\pi: \mathbf{R}^{n} \rightarrow \mathbf{R}^{n} / G$ is a differentiable fibre bundle. By using invariant polynomials it is possible to differentiably imbed $\mathbf{R}^{n} / G$ into some Euclidean space. By means of such an imbedding we can define the space of rectifiable currents $R_{*}\left(R^{n} / G\right)$ on $R^{n} / G$. Such a definition is both 
natural and imbedding invariant. For each $k$ we then define $\hat{I}_{k}\left(\mathbf{R}^{n} / G\right)=\{T \epsilon$ $\left.R_{k}\left(\mathbf{R}^{n} / G\right): \partial T L X / G \in \Re_{k \rightarrow 1}\left(\mathbf{R}^{n} / G\right)\right\}$.

For complete details and a number of examples related to the discussion in the above two paragraphs we refer the reader to [8]. We do include one example here which will be discussed more deeply later on.

Example 4.2. Let $G=S O(r) \times S O(s)$ acting on $\mathbf{R}^{r} \times \mathbf{R}^{s}$ in the standard way, where $r, s>1$. Then the principal orbits are all diffeomorphic to $s^{r-1} \times S^{s-1}$, and $X=\left\{(x, y) \in \mathbf{R}^{r} \times \mathbf{R}^{s}:|x| \cdot|y| \neq 0\right\}$. The orbit space can be represented as $\mathbf{R}^{r+s} / G=\left\{(u, v) \in \mathbf{R}^{2}: u \geq 0\right.$ and $\left.v \geq 0\right\}$ where $\pi: \mathbf{R}^{r+s} \rightarrow \mathbf{R}^{r+s} / G$ is given by $\pi(x, y)=(|x|,|y|)$. The canonical metric on $\mathbf{R}^{r+s} / G$ (compatible with the fibration over each stratum) is the usual flat one $d s^{2}=d u^{2}+d v^{2}$. The volume function is of course proportional to $V(u, v)=u^{r-1} v^{s-1}$.

We now consider a current $B=\partial \bar{T}$ where $\bar{T} \in I_{p+k}^{G}\left(\mathbf{R}^{n}\right)$ and where $\operatorname{supp}(B) C$ $X$. From the general compactness theorem for integral currents $[6,8.13]$ and the fact that $I_{*}^{G}\left(\mathbf{R}^{n}\right)$ is closed in $I_{*}\left(\mathbf{R}^{n}\right)$, we have

Proposition 4.3 (Existence). There exists an integral current $T \in I_{p+k}^{G}\left(\mathbf{R}^{n}\right)$ such that $\partial T=B$ and

$$
M(T)=\inf \left\{M\left(T^{\prime}\right): T^{\prime} \in I_{p+k}^{G}\left(R^{n}\right) \text { and } \partial T^{\prime}=B\right\} .
$$

We denote any such current $T$ as a solution to the equivariant Plateau problem for $B$.

We shall now formulate an equivalent Plateau problem in the orbit space $\mathrm{R}^{n} / G$ 。 In doing this the following fact will be crucial.

Proposition 4.4. Let $B=\partial \bar{T}$ where $\bar{T} \in I_{p+k}^{G}\left(\mathbf{R}^{n}\right)$ and $\operatorname{supp}(B) \subset X$, and let $\Re_{G, B}$ denote the set of $G \circ$ invariant, rectifiable, $(p+k)$-dimensional currents $T$ such that $\partial T=B+B^{\prime}$ where $\operatorname{supp}\left(B^{\prime}\right) \subset S$. Then for any $T \in R_{G, B}$ which satisfies $M(T)=\inf \left\{M\left(T^{\prime}\right): T^{\ell} \in \Re_{G, B}\right\}$ we have $\partial(T\llcorner X)=B$ 。

Proof. Since $S$ is a cone in $\mathbf{R}^{n}$ we have that $\operatorname{supp}(T)$ is contained in any ball centered at the origin which contains supp $(B)$. Thus we shall always work inside a compact ball. Note that since $T$ is minimal, $T=T\llcorner X$.

Let $f: \mathbf{R}^{n} \rightarrow \mathbf{R}$ be a Lipschitz function with Lipschitz constant 1 such that $f \mid X>0$ and $\inf \{f(x): x \in \operatorname{supp}(B)\}>\sup \{f(x): x \in S\}$. For each $\epsilon>0$, we let $X_{\epsilon}=$ $\left\{x \in \mathbf{R}^{n}: f(x)>\epsilon\right\}$ and set $T_{\epsilon}=T\left\llcorner X_{\epsilon^{\circ}}\right.$ By the dominated convergence theorem and 8.8 in [6] we have that

$$
\lim _{\epsilon \rightarrow 0} T_{\epsilon}=T_{0} \text { and } \quad \lim _{\epsilon \rightarrow 0} \partial T_{\epsilon}=\partial T_{0} .
$$

For $\epsilon$ sufficiently small, $\partial T_{\epsilon}=B+B_{\epsilon}^{\prime}$ where $\operatorname{supp}(B)$ and $\operatorname{supp}\left(B_{\epsilon}^{\prime}\right)$ are separated. Thus for small $\epsilon$,

$$
M\left(\partial T_{\epsilon}\right)=M(B)+M\left(B_{\epsilon}^{\prime}\right)
$$


Furthermore, $\lim _{\epsilon \rightarrow 0} B_{\epsilon}^{\prime}=B_{0}^{\prime}$, and, by the lower semicontinuity of mass,

$$
\varliminf_{\epsilon \rightarrow 0} M\left(B_{\epsilon}^{\prime}\right) \geq M\left(B_{0}^{\prime}\right) \text {. }
$$

Each $T_{\epsilon}$ is rectifiable. Hence by 8.14 in [6], $T_{\epsilon}$ is an integral current whenever $M\left(\partial T_{\epsilon}\right)<\infty$. By 3.10 in [6], we have $M\left(\partial T_{\epsilon}\right)<\infty$ for almost all $\epsilon$ in [0, $)$. In fact if $0 \leq a<b$ and $b$ is sufficiently small, then

$$
\int_{a}^{b} M\left(B_{\epsilon}^{\prime}\right) d \epsilon \leq M\left(T_{a}-T_{b}\right) .
$$

If we choose $f(x)=$ distance $(x, S)$, then $T_{0}=T$ and $B_{0}^{\prime}=B$. However, in general the singularities of $S$ make the proof that $\underline{\lim }_{\epsilon \rightarrow 0} M\left(B_{\epsilon}^{\prime}\right)=0$ quite difficult. Our procedure will therefore be to use functions which agree with this one except near the singularities of $S$.

We choose our function $f$ as follows. For any closed set $A \subset \mathbf{R}^{n}$ let $d_{A}(x)=$ distance $(x, A)$. The set $S$ is a homogeneous algebraic variety in $\mathbf{R}^{n}$ which has a subset $S^{\prime}$ of singular points. Let $\delta>0$ be a small number and let $\epsilon_{0}=$ $\max \left\{d_{S}(x): d_{S^{\prime}}(x) \leq \delta\right\}$. Set $\pi_{\delta}=\left\{x \in \mathbf{R}^{n}: d_{S^{\prime}}(x) \leq \delta\right\}$ and $S_{\epsilon_{0}}=\left\{x \in \mathbf{R}^{n_{0}}: d_{S}(x)=\epsilon_{0}\right\}_{\text {。 }}$ Then we define $f(x)$ by

$$
f(x)= \begin{cases}d_{S}(x) & \text { if } d_{s}(x) \geq \epsilon_{0}, \\ \epsilon_{0} & \text { if } d_{s^{\prime}}(x) \leq \delta, \\ \epsilon_{0}-d_{r_{\delta} \cup S_{\epsilon_{0}}}(x) & \text { otherwise. }\end{cases}
$$

Suppose now that $\underline{\lim }_{\epsilon \rightarrow 0} M\left(B_{\epsilon}^{\prime}\llcorner X)=a>0\right.$. Then from (4.7) we get the following. If $a<\infty$, then for any number $\alpha<1$ there exist arbitrarily small numbers $\epsilon>$ 0 such that $\epsilon a a \leq M\left(T_{0}-T_{\epsilon}\right)$. If $a=\infty$, then there exist arbitrarily small numbers $\epsilon$ such that

$$
\epsilon M\left(B_{\epsilon}^{\prime} L \quad X\right) \leq M\left(T_{0}-T_{\epsilon}\right) .
$$

Choose such an $\epsilon<<\epsilon_{0}$ and let $\Sigma=\left\{x \in \mathbf{R}^{n}: f(x)=\epsilon\right\}$. Observe that from our definition of $f$ the set $\left\{x_{0} f(x)>\epsilon\right\}$ contains $\boldsymbol{\gamma}_{\delta^{\circ}}$ Thus, $\Sigma$ is bounded away from $S^{\prime}$. Moreover, by choosing $\epsilon$ sufficiently small and using compactness, we may assume that $\Sigma$ lies in a neighborhood of the manifold $S \sim S^{\prime \prime}$, in which every point can be joined to $S \sim S^{\prime}$ by a unique, shortest straight line. It follows that the vector field $d_{S} \cdot \nabla d_{S}=1 / 2 \nabla\left(d_{S}^{2}\right)$ is well defined and differentiable in this neighborhood. We now let $F: \Sigma \times[0,1] \rightarrow \mathbf{R}^{n}$ be the map which deforms $\Sigma$ uniformly along the gradient of $d_{S}$. That is,

$$
F(x, t)=x-t d_{S}(x) \cdot \nabla d_{S}(x) .
$$

Let $Z=F_{H}\left(B_{\epsilon}^{\prime}\llcorner X \times I)\right.$. Then for any partition $t_{0}=0<t_{1}<t_{2}<\cdots<t_{N}=1$ we have 


$$
Z=\sum_{k=1}^{N} F_{\#}\left(B_{\epsilon}^{\prime} L \quad X \times\left[t_{k-1}, t_{k}\right]\right)
$$

and

$$
M(Z) \leq \sum_{k=1}^{N} M\left[\left(F_{t_{k}^{\prime}}\right)_{\#}\left(3_{\epsilon}^{\prime} L X\right)\right]_{\epsilon}\left(t_{k}-t_{k-1}\right),
$$

where $F_{t}(x)=F(x, t)$ and $t_{k-1} \leq t_{k}^{\prime} \leq t_{k}$. We now claim that, for each $k$,

$$
M\left[\left(F_{t_{k}^{\prime}}\right)_{H}\left(B_{\epsilon}^{\prime}\llcorner X)\right]<(3 / 2) t_{k} M\left(B_{\epsilon}^{\prime}\llcorner X) .\right.\right.
$$

To see this observe first that the deformation $F$ is $G$-invariant and consequently projects to a similar deformation $\widetilde{F}$ on $\mathbf{R}^{n} / G$. Introduce the standard Riemannian structure $\langle\cdot, \cdot\rangle$ on $\mathbf{R}^{n} / G$. Then by choosing $\epsilon$ sufficiently small we can assume $\left\|\left(\tilde{F}_{t}\right)_{\sharp}\right\|<3 / 2$ (in fact, $<1+\gamma$ for any fixed $\gamma>0$ ) for $0 \leq t \leq 1$. It is not difficult to see that for small $\epsilon$ we have $\left\|\left(F_{t}\right)_{\#}\right\|<3 / 2$, and the corresponding statement for $\left\|\left(\tilde{F}_{t}\right)_{\#}\right\|$ follows by restricting to vectors perpendicular to the orbits. The inequality (4.8) can now be deduced from the fact that the volume function must vanish at least to first order on $S$, and the mass of any invariant current $T$ in $X$ can be expressed as the integral of the volume function over $\pi_{*} T$ in $X / G$ (with metric $\langle\cdot, \cdot\rangle)$.

Putting together (4.8) and (4.9) and passing to a limit, we have

$$
M(\neg) \leq \int_{0}^{1} \frac{3}{2} \epsilon t M\left(3_{\epsilon}^{\prime}\llcorner X) d t=\frac{3}{4} \epsilon M\left(B_{\epsilon}^{\prime}\llcorner X) .\right.\right.
$$

In the case $a<\infty$, a similar argument shows that for appropriate choices of $\epsilon$ and $\alpha$ we have $M(Z) \leq 3 / 4 \epsilon \alpha a$.

We now let $\tau=T_{\epsilon}+Z$. Then $\partial \tau=B+\mathscr{B}$ where supp $\mathscr{B} \subset S$. However, $M(T) \leq$ $M\left(T_{\epsilon}\right)+M(Z)<M\left(T_{\epsilon}\right)+M\left(T_{0}-T_{\epsilon}\right)=M\left(T_{0}\right) \leq M(T)$. This contradicts minimality, and therefore

$$
\varliminf_{\epsilon \rightarrow 0} M\left(B_{\epsilon}^{\prime} L X\right)=0 .
$$

Since $\delta$ was arbitrary, we have that $\operatorname{supp}\left(B^{\prime}\right) \subset S^{\prime}$ 。

The set $S^{\prime}$ is again a homogeneous algebraic variety, and with $S$ replaced by $S^{\prime}$ the entire argument can be repeated. In a finite number of steps this shows that $B^{*}=0$ and the proof is complete.

Note. In almost any specific case much shorter proofs of Proposition 4.4 can be given. The variety of difficulties presented by different group actions makes the above general argument somewhat involved.

Proposition 4.4 says that finding currents of least mass in $R_{G, B}$ is the same as solving the equivariant Plateau problem for $B$. However, under the map $\pi_{*}$ the 
currents in $R_{G, B}$ of least mass correspond to currents in $\hat{I}_{k}\left(R^{n} / G\right)$ of least $M_{k}$. mass having boundary $\pi_{*} B+B^{0}$ with supp $\left(B^{\prime}\right) \subset S / G$. Thus we have the following.

Theorem 3. Let $B=\partial \bar{T}$ where $\bar{T} \in I_{p+k}^{G}\left(\mathbf{R}^{n}\right)$ and supp $(B) \subset X$. Then there is a natural one-to=one correspondence $\phi_{:} \mathcal{S}^{\underline{b}} \rightarrow \mathcal{S}$ between the set $\mathcal{S}^{G}$ of solutions to the equivariant Plateau problem for $B$ in $\mathbf{R}^{n}$ and the set $\mathcal{S}$ of currents $T \epsilon$ $\hat{I}_{k}\left(\mathbf{R}^{n} / G\right)$ with $\partial T=\pi_{*}(B)+B^{\prime}$, where $\operatorname{supp}\left(B^{\prime}\right) \subset S / G$, and with $M_{k}(T)=$ $\inf \left\{M_{k}\left(T^{\prime}\right): T^{\prime} \in I_{k}\left(\mathbf{R}^{n} / G\right) \text { and } \partial T^{\prime}=\pi_{*} B+B^{\prime \prime} \text {, where supp }\left(B^{\prime \prime}\right) \subset S / G\right\}_{\text {。 In fact, }}$

$$
\phi(T)=\pi_{*}\left(T\llcorner X) \quad \text { and } \quad \phi^{-1}(T)=\pi^{*}(T\llcorner X / G) .\right.
$$

Remark 4.5. The condition that there are no nonprincipal orbits of dimension $p$ can be eliminated in the above work by including such orbits in $X$, defining the volume function as in [8], and using arguments which involve the differentiable slice theorem. Invariant manifolds passing through such orbits always have singularities even though their images in $X / G$ are regular. Consider, for example, the action of $S O(2)$ on $\mathbf{R}^{4}=\mathbf{C}^{2}$ which maps $(z, w)$ to $\left(e^{i m \theta} z, e^{i n \theta} w\right)$ for $\theta \in \mathbf{R}$ and $(m, n)=1$.

Theorem 3 is of particular interest when $k=1$. In this case the equivariant Plateau problem is reduced, after applying the regularity results in [2] and standard arguments concerning the regularity of piecewise smooth arcs of minimum length, to finding certain shortest geodesics in the manifold $X / G$ with metric $\langle\cdot, \cdot\rangle_{1}$. If, moreover, $n=p+2$ and the boundary $B$ lies on a $G$-invariant, convex hypersurface in $\mathbf{R}^{n}$, then by Theorem 1 , solutions to the equivariant problem are solutions to the general Plateau problem, and uniqueness for the geodesic(s) implies uniqueness for the general problem.

Consider, for instance, the case $r=1$ and $s=p+1$ in Example 4.2. Here the orbit space is

$$
\mathbf{R}^{p+2} / S O(p+1) \cong\left\{(u, v) \in \mathbf{R}^{2}: v \geq 0\right\},
$$

where $\pi: \mathbf{R}^{1} \times \mathbf{R}^{p+1} \rightarrow \mathbf{R}^{p+2} / S O(p+1)$ is $\pi(x, y)=(x,|y|)$, and the metric $\langle\cdot, \cdot\rangle_{1}$ is $d s^{2}=v^{2 p}\left(d u^{2}+d v^{2}\right)$. The geodesics for this metric are all of the form

$$
u=c \int_{a}^{v} \frac{d v}{\left(v^{2 p}-c^{2}\right)^{1 / 2}}
$$

for constants $c$ and $a$. When $p=1$, these are the lines $u=$ constant and $v=$ $c \cdot \cosh \left(u / c+a^{\prime}\right)$ which correspond respectively to planes (or disks) and the classic catenoids in $\mathbf{R}^{3}$. If we consider an invariant boundary in $\mathbf{R}^{p+2}$ of the type type $S^{p} \cup S^{p}$ (with opposite orientations on the components), then the correspond. ing boundary in $\mathbf{R}^{p+2 / S O}(p+1)$ is a pair of points. The shortest geodesic $\gamma$ joining thes? points (modulo the boundary) is either a generalized catenary (given by (4.10)) or a pair of vertical lines. The inverse image of $\gamma$ under $\pi$ is a solution 
to the general Plateau problem in $\mathbf{R}^{p+2}$ and except when the length of the "cato enary" equals the length of the two vertical lines it is the unique solution.

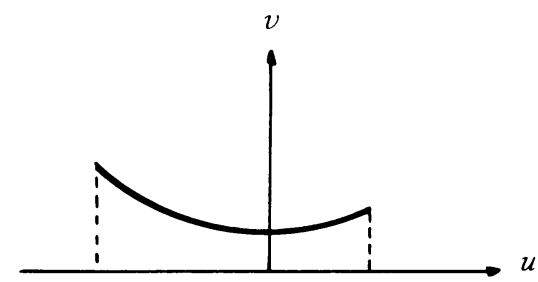

5. Interior regularity and uniqueness for the Plateau problem. One situation to which the theorems proved above have immediate relevance is the following. Consider the Plateau problem in $\mathbf{R}^{n+1}$ for an $(n-1)$-dimensional boundary manifold $M$ which can be realized as a (principal) orbit of an orthogonal representation of a compact, connected group $G$ on $R^{n+1}$. By Theorem 1 every solution to the $G$-invariant Plateau problem is a solution to the general Plateau problem, and uniqueness in the former case implies uniqueness in general. Furthermore by Theorem 3, solutions to the equivariant problem correspond naturally to solutions of the reduced problem in $\mathbf{R}^{n+1} / G$. The linear groups producing codimension=2 principal orbits on $\mathbf{R}^{n+1}$, and the associated orbit spaces with their natural metrics and volume functions are classified in [8], and listed here in the appendix. Each such orbit space can be realized isometrically as the cone over an arc of the unit circle in $\mathbf{R}^{2}$, and the square of the volume function, $V^{2}$, is a homogeneous polynomial in $x$ and $y$. The boundary manifold $M$ corresponds now to a point $m \in$ int $\left(\mathbf{R}^{n+1} / G\right) \subset \mathbf{R}^{2}$, and a solution to the reduced Plateau problem will be $a$ geodesic of minimum length in the metric

$$
d s^{2}=V^{2}\left(d x^{2}+d y^{2}\right),
$$

which connects $m$ to the boundary of the cone $\mathbf{R}^{n+1} / G$ in $\mathbf{R}^{2}$.

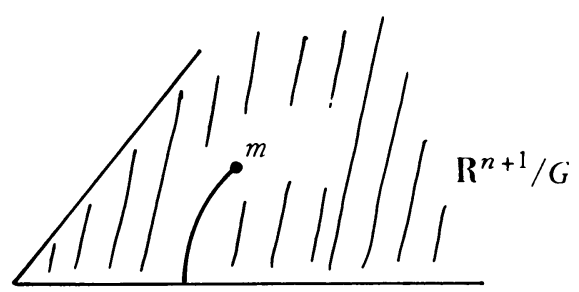

In all such cases, if the minimizing geodesic meets a wall of the cone, it meets it orthogonally, and the corresponding solution in $\mathbf{R}^{n+1}$ is a regular, imbed. ded, analytic manifold.

If the minimizing geodesic meets the vertex of the cone, of course the corresponding solution is singular. However, it can be shown, in all cases, that the 
only geodesic which meets the vertex is the straight line passing through a point $m_{0}$ on the unit circle where $m_{0}$ corresponds to the orbit of greatest volume on the unit sphere in $\mathbf{R}^{n+1}$. This line represents a cone in $\mathbf{R}^{n+1}$ over a minimal submanifold of the sphere. To show non-interior-regularity for solutions to problems of this sort we must therefore show that the geodesic line $\overline{0 m_{0}}$ is the arc of minimum length joining $m_{0}$ to the boundary of $\mathbf{R}^{n+1} / G$.

Many of the orbit spaces (of linear actions with codimension-2 orbits) listed in Table 1 have the following form:

$$
\mathbf{R}^{n+1} / G \simeq\{z \in \mathrm{C}: 0 \leq \arg z \leq \pi / p\} \text { with metric } d s^{2}=\operatorname{Im}\left\{Z^{p}\right\}^{q}|d z|^{2} .
$$

In this case $m_{0}=e^{i \pi / 2 p}$. We shall now establish an elementary criterion for determining when the line $\gamma_{0}=\left\{t_{0}: 0 \leq t \leq 1\right\}$, is the unique minimizing geodesic.

By letting $w=z^{p}$ we can replace the above orbit space by

$$
H=\left\{w \in \mathbf{C}: \mathfrak{T}_{m}(w) \geq 0\right\} .
$$

Choosing polar coordinates $w=r e^{i \theta}$ the metric (5.2) can be written

$$
d s^{2}=r^{2 a-2} \sin ^{q} \theta\left(r^{2} d \theta^{2}+d r^{2}\right),
$$

where

$$
2 \alpha-2=q-(2 p-2) / p
$$

It is sufficient for our purpose to find a function $f: H \rightarrow \mathbf{R}$ of class $C^{1}$ in $H^{0}$ such that

$$
\begin{gathered}
f_{r}^{2}+r^{-2} f_{\theta}^{2} \leq r^{2 a_{-2}} \sin ^{a} \theta, \\
f_{r}^{2}(r, \pi / 2)=r^{2 a_{-}},
\end{gathered}
$$

since then the closed differential form $d f=f_{r} d r+(1 / r) f_{\theta} r d \theta$ has the property that

$$
\text { length }\left(\gamma_{0}\right)=\int_{\gamma_{0}} d f=\int_{\gamma} d f \leq \text { length }(\gamma)
$$

for any curve $\gamma$ joining $m_{0}$ to the boundary. To see that the integrals in (5.7) are equal one approximates $\gamma_{0}-\gamma$ by closed curves as shown in Figure 5.1 and notes that length $\left(\gamma_{i}\right) \rightarrow 0$ as $i \rightarrow \infty$.

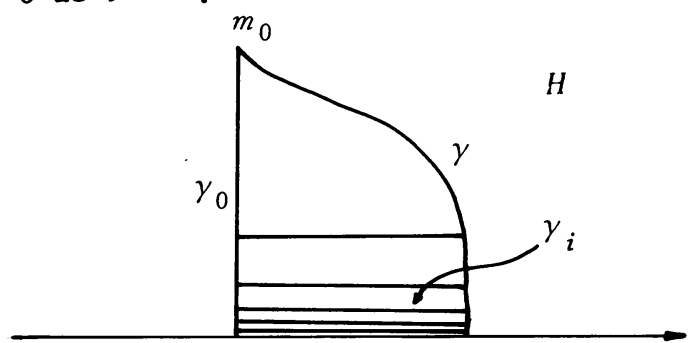

Figure 1 
We note that if equality in (5.5) holds only when $\theta=\pi / 2$, then equality in (5.7) will hold only when $\gamma=\gamma_{0}$, and uniqueness will be established. We try a function $f$ of the type $f=\alpha^{-1} r^{\alpha} \sin ^{\beta} \theta$. Here (5.6) is satisfied for all $\beta$ and $(5.5)$ is reduced to the inequality

$$
\sin ^{2 \beta} \theta+(\beta / \alpha)^{2} \sin ^{2 \beta-2} \theta \cos ^{2} \theta \leq \sin ^{q} \theta
$$

Thus, we want the function

$$
\phi(\theta) \stackrel{\text { def }}{=} 1-\sin ^{2 \beta-q} \theta-(\beta / \alpha)^{2} \sin ^{2 \beta-2-q} \theta \cos ^{2} \theta \geq 0
$$

for $0 \leq \theta \leq \pi$ and for some choice of $\beta$ where

$$
\beta>1+q / 2
$$

For fixed $\beta$ this is guaranteed if

$$
\phi^{\prime}(\theta)=0 \text { only when } \theta=\pi / 2
$$

(since $\phi(\pi / 2)=0$ and $\phi(0)=\phi(\pi)=1$ ). However, by computing $\phi^{\prime}(\pi)$ one finds (5.9) equivalent to the condition:

$$
a^{2}(2 \beta-q)-2 \beta^{2}>0 .
$$

The expression in $(5.10)$ reaches a maximum when $\beta=\alpha^{2} / 2$. Thus, substituting (5.4) in (5.8) and $(5.10)$ with $\beta=\alpha^{2} / 2$, we get the following.

Proposition 5.1. In an orbit space of type (5.2) the geodesic $\gamma_{0}$ is the unique curve of shortest length joining $m_{0}$ to the boundary if

(a) $q^{2} / 4+q / p+1 / p^{2}>q+2$ and

(b) $q^{2}-(8-4 / p) q+4 / p^{2}>0$ 。

Checking the list of metrics in Table 1 we find that (a) and (b) are satisfied in cases (1) where $r=s \geq 3,(5),(8),(11)$, and (12). Thus, we have

Theorem 4. There exist manifolds $M$ of type $S^{n} \times S^{n}$ in $\mathbf{R}^{2 n+2}$ for $n \geq 3$, $S_{p}(3) /\left(S_{p}(1)\right)^{3}$ in $\mathbf{R}^{14}$, SU(5)/SU(2) $\times S U(2)$ in $\mathbf{R}^{20}, F_{4} / S_{p i n}(8)$ in $\mathbf{R}^{26}$ and Spin(10)/SU(4) in $\mathbf{R}^{32}$ such that a cone over $M$ represents the unique integral current of least mass baving $M$ as boundary. In particular, the bope for interior regularity of solutions to the Plateau problem fails in dimension eight.

We now apply a similar argument to the remaining cases in Table 1. In these cases the orbit space can be written as

$$
Q=\left\{(x, y) \in \mathbf{R}^{2}: x \geq 0 \text { and } y \geq 0\right\}
$$

where the metric is

$$
d s^{2}=\left(x^{p} y^{q} / r^{l}\right)\left(d x^{2}+d y^{2}\right)
$$


for $l=0$ in case (1) and $l=1, p=2,4$ or 8 in cases (2), (3) or (4) respectively.

As before we choose polar coordinates and search for a function $f \in C(Q) \cap$ $C^{1}\left(Q^{0}\right)$ such that

(i) $f_{r}^{2}+r^{-2} f_{\theta}^{2} \leq r^{p+q+l} k-1 \cos ^{p} \theta \sin ^{q} \theta$,

(ii) $f_{r}^{2}\left(r, \theta_{0}\right)=r^{p+q+l}$,

where $\tan ^{2} \theta_{0}=q / p$ and $k=\cos ^{p} \theta_{0} \sin ^{q} \theta_{0}$. Let

$$
\phi=k^{-1} \cos ^{p} \theta \sin ^{q} \theta
$$

and observe that $\phi^{\prime}(\theta)=\phi(\theta)(q \operatorname{ctn} \theta-p \tan \theta)$. We try a solution of the type $f=a^{-1} r^{a} \phi^{\beta}$ where

$$
2 a=p+q-l+2,
$$

and we are reduced to the inequality

$$
\psi(\theta) \stackrel{\text { def }}{=} 1-\phi^{2 \beta-1}(\theta)\left[1+(\beta / \alpha)^{2}(q \operatorname{ctn} \theta-p \tan \theta)^{2}\right] \geq 0 .
$$

Since we need to have $\phi^{2 \beta-1} / \sin ^{2} \theta \cos ^{2} \theta \rightarrow 0$ as $\theta \rightarrow 0$ and $\theta \rightarrow \pi / 2$, we must require that

$$
\beta>1 / 2+\max (1 / p, 1 / q) \text {. }
$$

Under this assumption we have that $\psi(0)=\psi(\pi / 2)=1$ and $\psi\left(\theta_{0}\right)=0$. Thus, it suffices to prove that $\psi^{\prime}(\theta)=0$ only at $\theta=\theta_{0}$ for some choice of $\beta$ satisfying (5.12). Computing, we find that $\psi^{\prime}(\theta)=0$ only at $\theta=\theta_{0}$ if and only if

$$
F(\theta) \stackrel{\text { def }}{=} A\left[(\alpha / \beta)^{2}-(p+q)^{2}\right] \sin ^{2} \theta \cos ^{2} \theta+\left(A p^{2}-p\right) \sin ^{2} \theta+\left(A q^{2}-q\right) \cos ^{2} \theta \geq 0
$$

for $0 \leq \theta \leq \pi / 2$, where

$$
A=\beta-1 / 2 \geq \operatorname{nax}(1 / p, 1 / q) .
$$

Note that $F(0)>0, F(\pi / 2)>0$ and that the coefficient of $\cos ^{2} \theta \sin ^{2} \theta$ in $F$ is $<0$ for all acceptable $\beta$. We then compute the minimum value of $F$ to be

$$
\begin{gathered}
2 F_{\min }=A\left[(\alpha ; \beta)^{2}-(p+q)^{2}\right]+2\left[A\left(p^{2}+q^{2}\right)-(p+q)\right] \\
+\frac{\left[A\left(p^{2}-q^{2}\right)-(p-q)\right]^{2}}{A\left[(\alpha / \beta)^{2}-(p+q)^{2}\right]} .
\end{gathered}
$$

Letting $\sigma=p+q$ and $d=p-q$ we can rewrite this as

$$
2 F_{\min }=A\left[(\alpha / \beta)^{2}+d^{2}\right]-2 \sigma+\frac{d^{2}(A \sigma-1)^{2}}{A\left[(\alpha / \beta)^{2}-\sigma^{2}\right]} .
$$

We now let $l=0$ (corresponding to case (1)) and choose $2 \beta=(\sigma+2)^{2} / 8 \sigma$. (Thus, $\left.A=(\sigma-2)^{2} / 16 \sigma.\right)$ Then, $F_{\min }>0$ if and only if 


$$
\left(\sigma^{2}-12 \sigma+4\right)\left[1-\alpha \frac{d^{2}}{\sigma^{2}}\right]>8 \alpha \frac{d^{2}}{2}\left(\frac{\sigma+2}{\sigma-2}\right)^{2},
$$

where $a=(\sigma+2)^{2} /\left((\sigma+2)^{2}-64\right)$. When $d=0$ we get the previous result, but more generally we now have the following.

Theorem 5. For $r+s \geq 10$, or $r+s=9$ and $|r-s| \leq 5$, or $r+s=8$ and $|r-s| \leq 1$, the cone over the manifold

$$
s^{r-1} \times s^{s-1}=\left\{(x, y) \in \mathbf{R}^{r} \times \mathbf{R}^{s}:|x|^{2}=r /(r+s) \text { and }|y|^{2}=s /(r+s)\right\}
$$

with respect to the origin is the unique minimizing integral current in $R^{r+s}$ baving $S^{r-1} \times S^{s-1}$ as boundary.

If we let $l=1$ (corresponding to cases (2), (3) and (4)) and choose $2 \beta=$ $(\sigma+1)^{2} / 8 \sigma$, we find that $F_{\min }>0$ if and only if

$$
\left(\sigma^{2}-14 \sigma+1\right)\left[1-\alpha \frac{d^{2}}{\sigma^{2}}\right]>8 \alpha \frac{d^{2}}{\sigma^{2}} \frac{(\sigma+1)^{2}}{\sigma^{2}-6 \sigma+1},
$$

where $\alpha=(\sigma+1)^{2} /\left((\sigma+1)^{2}-64\right)$. We then have that there are minimizing cones over manifolds of type $S O(2) \times S O(k) / \mathbf{Z}_{2} \times S O(k-2)$ in $\mathbf{R}^{2 k}$ for $k \geq 10$, of type $S U(2) \times S U(k) / T^{1} \times S U(k-2)$ in $\mathbf{R}^{4 k}$ for $k \geq 5$, and of type $S_{p}(2) \times S p(k) / S_{p}(1)^{2}$ $\times S p(k-2)$ in $\mathbf{R}^{8 k}$ for $k \geq 2$.

The reason for the non-interior-regularity of certain minimizing (codimensionone) minimal currents in large dimensions is the high order of vanishing of volume functions at the origin. For the actions given in Example 4.2 with $r=s=n+1$ the orbit space is $\left\{(u, v) \in \mathbf{R}^{2}: u \geq 0\right.$ and $\left.v \geq 0\right\}$ and the metric is

$$
a s^{2}=u^{2 n} v^{2 n}\left(d u^{2}+d v^{2}\right)
$$

For any $n$ there are just two possible (noncongruent) geodesics which could be the shortest curve joining the point $(1,1)$ to the boundary as shown in the figure. For $n=1,2$ the curve $\gamma_{1}$ (or its mirror image) is the shortest. However, as seen in (5.11), for large $n$ the volume function shrinks the orbit space along the diagonal much more that it does elsewhere, and for $n \geq 3$ the diagonal line $\gamma_{0}$ is in fact the shortest route to the boundary.

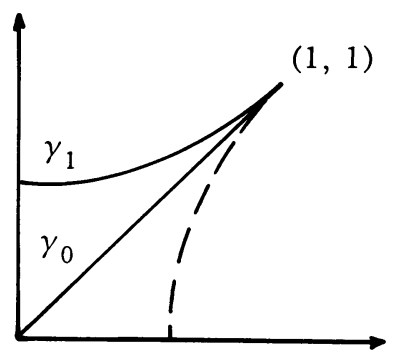




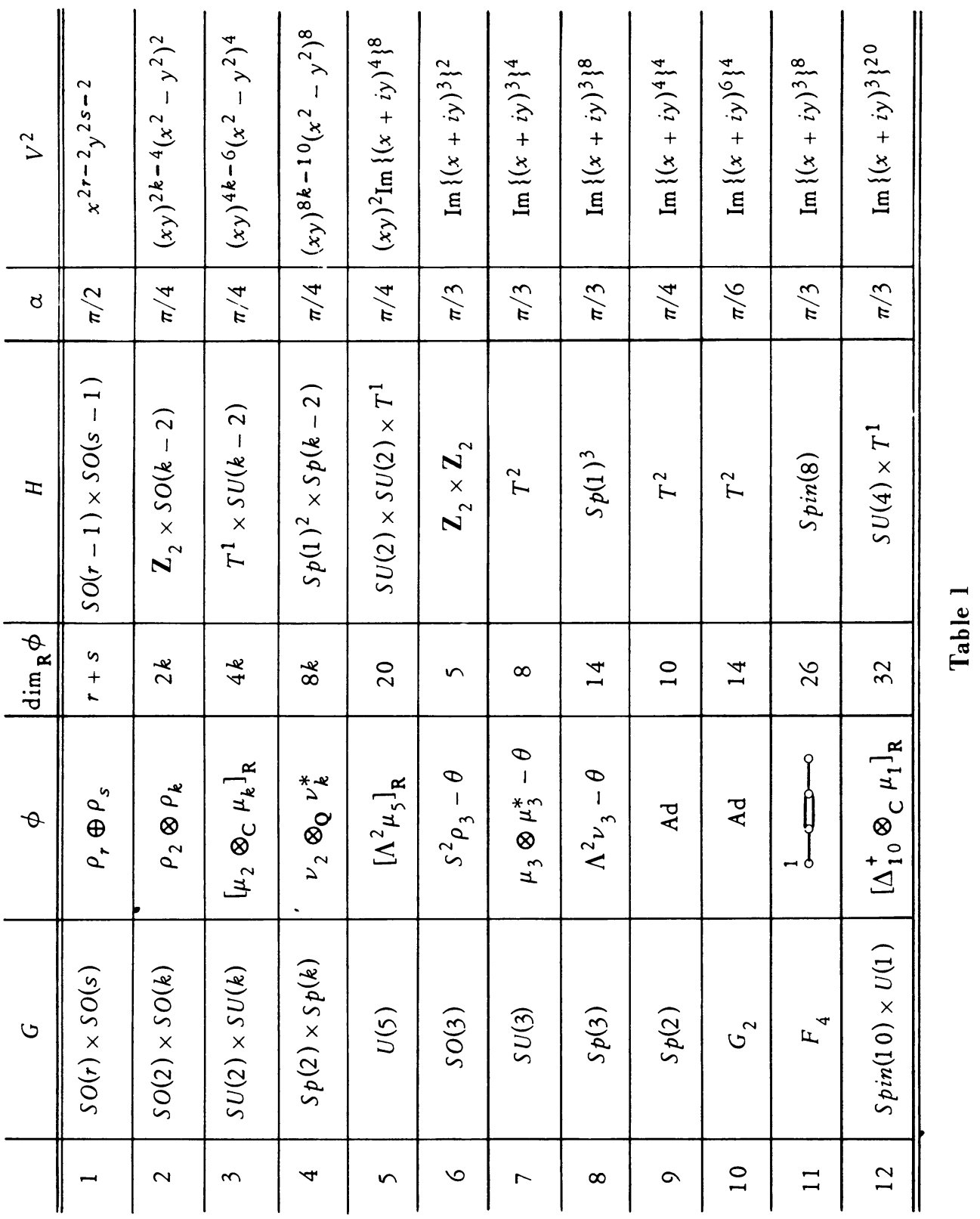


Appendix: Linear groups with codimension-two principal orbits. The following is a complete list of the compact, connected Lie groups $G$ with orthogonal representations $\phi$ which produce principal orbits of codimension two. This is a classification of orbit structures. Thus, if $\left(G_{1}, \phi_{1}, \mathbf{R}^{n}\right)$ and $\left(G_{2}, \phi_{2}, \mathbf{R}^{n}\right)$ have the same orbit structure, only the larger group is mentioned.

The notation for the table is defined as follows. $H$ is the principal isotropy subgroup, and the principal orbits are, therefore, diffeomorphic to $G / H$. The orbit space is represented isometrically as

$$
C(\alpha)=\left\{(x, y) \in \mathbf{R}^{2}: 0 \leq \operatorname{Tan}^{-1}(y / x) \leq \alpha \text { and } x \geq 0\right\}
$$

and $V=V(x, y)$ denotes the volume function. The metric appropriate to the codi. mension-one Plateau problem is then $d s^{2}=V^{2}\left(d x^{2}+d y^{2}\right)$. The symbols $\rho_{n}, \mu_{n}$ and $\nu_{n}$ denote the standard representations of $S O(n), S U(n)$ and $S_{p}(n)$ on $\mathbf{R}^{n}$, $\mathbf{R}^{2 n}$ and $\mathbf{R}^{4 n}$ respectively. The symbol $\theta$ represents the one-dimensional trivial representation. The reference for this table is [8].

\section{BIBLIOGRAPHY}

1. W. Allard, Boundary regularity for the Plateau problem, Ph. D. Thesis, Brown University, Providence, Rhode Island, 1968.

2. F. J. Almgren, Jr., Existence and regularity almost everywhere of solutions to elliptic variational problems among surfaces of varying topological type and singularity structure, Ann. of Math. (2) 87 (1968), 321-391. MR 37 \#837.

3. E. Bombieri, E. DeGiorgi and E. Guisti, Minimal cones and the Bernstein problem, Invent. Math. 7 (1969), 243-268. MR $40 \# 3445$.

4. J. Brothers, Integral geometry in homogeneous spaces, Trans. Amer. Math. Soc. 124 (1966), 480-517. MR 34 \#1973.

5. H. Federer, Geometric measure theory, Die Grundlehren der math. Wissenschaften, Band 153, Springer-Verlag, New York, 1969. MR 41 \#1976.

6. H. Federer and W. H. Fleming, Normal and integral currents, Ann. of Math. (2) 72 (1960), 458-520. MR 23 \#A588.

7. H. Federer, The singular sets of area minimizing rectifiable currents with codimension one and of area minimizing flat chains modulo two with arbitrary codimension, Bull. Amer. Math. Soc. 76 (1970), 767-771. MR 41 \#5601.

8. W. Y. Hsiang and H. B. Lawson, Minimal submanifolds of low cohomogeneity, J. Differential Geometry 5 (1971), 1-38.

9. B. O'Neill, The fundamental equations of a submersion, Michigan Math. J. 13 (1966), 459-469. MR $34 \# 751$.

10. E. R. Reifenberg, On the analyticity of minimal surfaces, Ann. of Math. (2) 80 (1964), 15-21. MR 30 \#1429.

DEPARTMENT OF MATHEMATICS, UNIVERSITY OF CALIFORNIA, BERKELEY, CALIFORNIA 94720

Current address: School of Mathematics, Institute for Advanced Study, Princeton, New Jersey 08540 